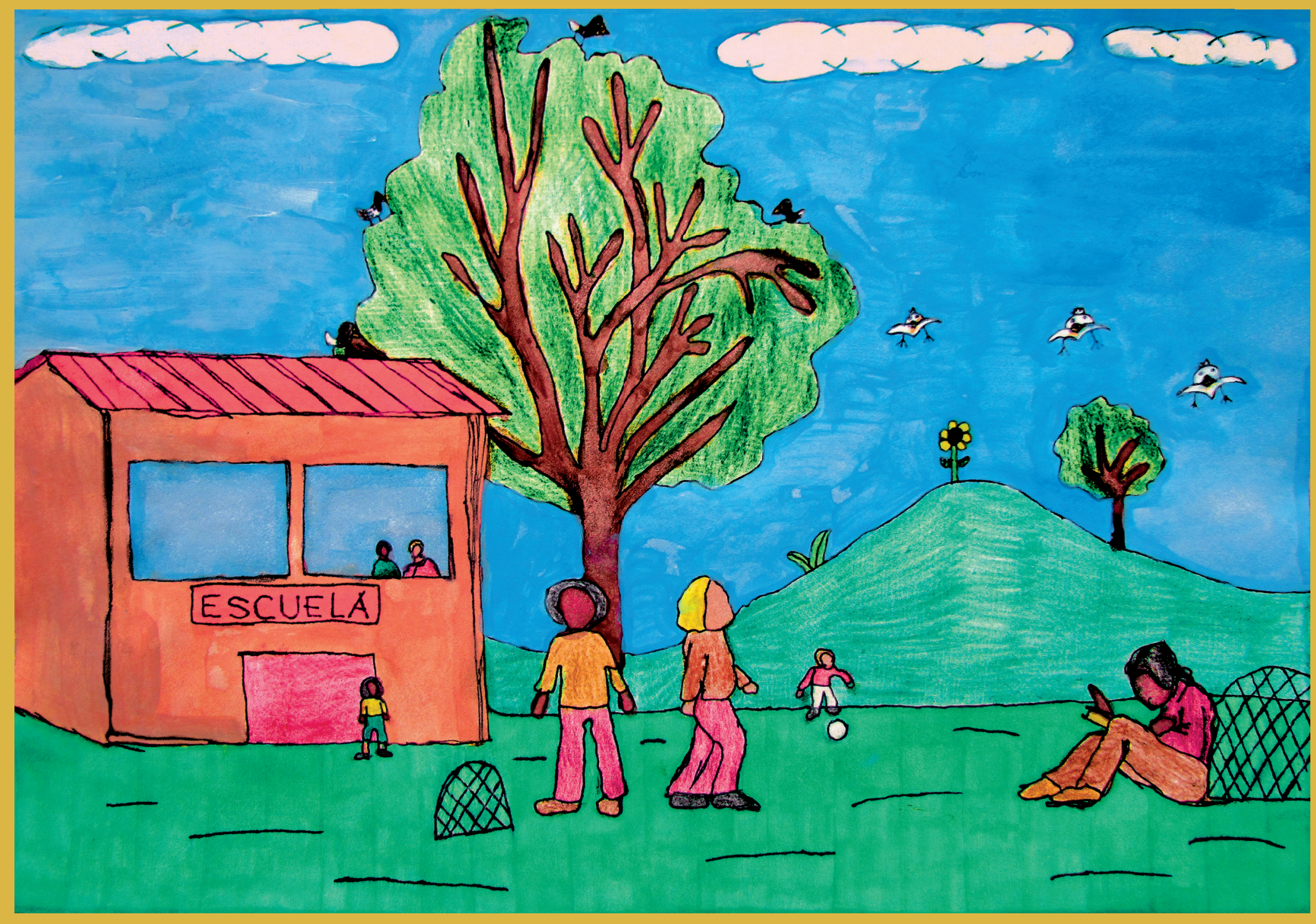

Clara Eugenia Bahamón - Arte sin Fronteras - Programa Talentos Especiales

Gloria Isabel Bermudez Jaimes

Magister en Discapacidad e Inclusión social

isabelbermudezjaimes@gmail.com

Claudia Carolina Bayona González

Magister en Lingüística

ccbayonag@yahoo.es

ESCUELA COLOMBIANA DE REHABILITACIÓN

Grupo de investigación Comunicación y diversidad 


\section{DISEÑO DE UNA PRUEBA TAMIZ PARA CARACTERIZAR PRERRECURRENTES DE LECTURA Y ESCRITURA EN POBLACIÓN PREESCOLAR}

\section{Screening Test Design to Characterize the Early Literacy of Reading and Writing in Preschool Population}

Fecha de Recepción: 21 de Agosto de 2011 - Fecha de Aprobación: 10 de Octubre de 2011

\section{RESUMEN}

El estudio se propuso diseñar una prueba tamiz para caracterizar los prerrecurrentes de lectura y escritura en población preescolar de 2 a 5 años de edad. Para la elaboración del instrumento se siguió el método propio del diseño y construcción de pruebas. Para la fase de conceptualización se consultaron 38 fuentes bibliográficas y 17 baterías de evaluación relacionadas con el tema de aprendizaje de la lectura, la escritura y el desarrollo del lenguaje. Para la fase de ensayo de los reactivos se recurrió a la validación por juicio de 8 expertos, y a la realización de una prueba piloto en una población de 50 niños y niñas del rango de edad requerido. Como resultado se obtuvo un instrumento que permite identificar comportamientos, conocimientos, habilidades y destrezas de tipo cognitivo, metacognitivo, lingüístico y actitudinal que ejecutan los niños y que se consideran precursores para el aprendizaje y adecuado desarrollo de la lectura y la escritura.

\section{PALABRAS CLAVE}

Prerrecurrentes de lectura, prerrecurentes de escritura, desarrollo de la lectura, desarrollo de la escritura, prueba tamiz de lectura y escritura

\section{ABSTRAC}

The objective of this study was to design a screening test to characterize the early literacy of reading and writing in pre-school population from 2 to 5 years of age. The own method of the design and construction of tests were taken in order to produce the instrument. For the conceptualization phase, 38 literature sources were consulted and 17 batteries of assessment related to the topic of learning of reading, writing and language development. For the testing phase of the reagents, for validation was resorted by judgment of 8 experts, and to the accomplishment of a pilot test in a population of 50 boys and girls with the range of range required age. As a result, got to build an instrument that allows to identify the occurrence of knowledge. abilities and cognitive metacognitive, linguistic and behavioural skills, that early literacy is considered appropriate for the learning and suitable development of the reading and the writing, and which are in normal conditions in early childhood

KEY WORDS

Early literacy, Reading, Writing, Development, Screening test 
INTRODUCCIÓN

L a lectura y la escritura son actividades complejas que implican altas demandas cognitivas, lingüísticas, motoras, sensoperceptivas y afectivas para su aprendizaje. Dicho aprendizaje también está influenciado por la interacción del niño en escenarios y prácticas sociales que promueven o dificultan el desarrollo de los

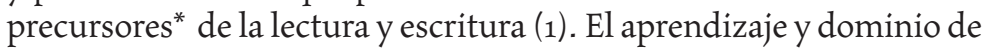
la lectura y la escritura es un proceso que se sustenta en habilidades propias de los primeros años de vida del niño conocidas como prerrecurrentes o precursores de la lectura y la escritura y que se complejiza y perfecciona durante toda la vida (2).

Existe un amplio conjunto de investigaciones en las que se evidencia la relevancia de los primeros años de vida en el desarrollo integral del niño (3). De igual modo existen estudios que demuestran la importancia del ambiente social y familiar como favorecedor de la aparición y afianzamiento de las destrezas cognitivas, lingüísticas, motoras, sensoperceptivas y afectivas, previas para poder aprender la lectura y la escritura $(3,4)$. En ambientes tradicionales los niños reciben estos estímulos los primeros años de su vida en la interacción cotidiana con sus padres y adultos significativos antes del inicio de la edad escolar (1). Todas las experiencias cotidianas enriquecen estas habilidades y configuran los futuros desempeños de los niños como lectores y escritores, que posteriormente incidirán de forma positiva en su rendimiento académico (3).

Estos prerrecurrentes hacen parte del acervo de habilidades y destrezas que posee el niño y que configuran el desarrollo del lenguaje en los primeros años. Por este motivo, en la práctica pedagógica y clínica los profesionales se interesan por monitorear el estado del desarrollo del lenguaje como unidad central, de manera que la mayoría de test y pruebas de las que se valen estos profesionales se dirigen a la evaluación general del lenguaje (BLOC, PLON, BATTELLE, PLS, ELCE, GARDNER) (5-10). Al día de hoy son pocas, las baterías de evaluación que indagan por estas habilidades en el marco del proceso de aprendizaje de la lectura y la escritura (11), y además, éstas, en su mayoría se concentran en las habilidades lingüísticas exclusivamente $(6,8,10,12-19)$; otras pocas evalúan las habilidades metalingüísticas (20), pero ninguna observa las tres de manera simultánea.

La falta de instrumentos especializados para la valoración específica de los prerrecurentes de lectura y escritura dificulta el proceso de evaluación de estas habilidades en contextos específicos como el ambiente escolar, o en la práctica clínica cuando se atienden problemáticas asociadas a este tema, ya que, como se mencionó anteriormente, para evaluar en su totalidad estos prerrecurrentes el profesional debe aplicar varias pruebas o parte de ellas, lo cual demora y complejiza la evaluación. Contar con una batería para valorar el estado de los perrecurrentes de la lectura y la escritura facilita el proceso diagnóstico y ofrece una guía eficiente para los profesionales novatos o en formación que no tiene el criterio para seleccionar las pruebas y subpruebas de otras baterías para evaluar este mismo aspecto.
La prueba tamiz de prerrecurrentes de lectura y escritura que se propone en este estudio es una batería de evaluación cualitativa que permite identificar la presencia y/o ausencia de habilidades orales, escriturales y actitudinales que desarrollan los niños en los primeros años y que configuran sus futuros desempeños como lectores y escritores. La prueba retoma elementos de la psicología cognitiva, la psicolingüística, la psicogenética y el socio-constructivismo para lograr abordar de manera simultánea los elementos lingüísticos, metacognitivos y actitudinales que determinan estos prerrecurrentes tales como la conciencia fonológica, el conocimiento de lo impreso, el razonamiento verbal y la motivación para leer y escribir.

Contar con una nueva prueba para evaluar los prerrecurrentes de lectura y escritura en población preescolar permitirá iniciar estudios orientados a caracterizar estos prerrecurrentes en población colombiana, con el objetivo de establecer una base de evidencia científica respecto a la relación que existe entre el desarrollo de estos prerrequisitos y otras variables de interés para campos de estudio propios de la fonoaudiología, la psicología y la pedagogía, entre otros. Además, a partir de esta investigación se avanzará en el desarrollo de nuevas técnicas para valorar los prerrecurrentes de la lectura y la escritura; y establecer líneas de intervención para potencializar estos desarrollos en población en situación de vulnerabilidad social.

Antes de presentar los hallazgos de la investigación es importante mencionar los referentes teóricos y conceptuales que soportan este estudio.

La lectura y la escritura son actividades que implican la integración de múltiples procesos cognitivos de alto y bajo nivel (4). Su aprendizaje requiere que el niño posea un desarrollo integral apropiado. (3) La lectura y la escritura constituyen una creación cultural, puesto que los grupos sociales en su interés por preservar los conocimientos y tradiciones propios generaron un conjunto de símbolos arbitrarios que se establecieron de manera convencional por la comunidad cuyo propósito era representar la comunicación oral, este proceso requirió muchos años en su desarrollo y difusión (21, 22).

La lectura se define como un proceso lingüístico-cognitivo que implica: i) decodificar una serie de grafías y asignarles una correspondencia fonética; ii) configurar esas unidades fonémicas en unidades silábicas, palabras y frases con un significado. Por lo tanto, aprender a leer implica reconocer las palabras y comprender la información escrita $(4,23)$.

La escritura por su parte, es una actividad cognitivo-lingüísticamotora, en la que el sujeto codifica una serie de ideas mediante un conjunto de grafías, conformando silabas, palabras, frases y unidades discursivas mayores, como los textos. Como ocurre con la lectura el aprendizaje de la escritura implica el desarrollo de habilidades mecánicas, es decir, la correspondencia entre fonemas y grafías, así como habilidades ortográficas. Una vez se automatiza la codificación se emplean las habilidades cognitivas en el perfeccionamiento de las

\footnotetext{
* A lo largo del documento se emplearan los términos prerrecurrentes o precursores de manera indiferenciada para hacer referencia al conjunto de destrezas cognitivas, lingüísticas, motoras, sensoperceptivas y afectivas, previas al aprendizaje de la lectura y la escritura.
} 
habilidades de composición textual, proceso que puede realizarse a lo largo de toda la vida $(4,24)$.

Durante mucho tiempo las investigaciones sobre la lectura y la escritura se enfocaron en la metodología de su enseñanza. Sin embargo, con el auge de la psicología cognitiva, la psicolingüística, la antropología, la fonoaudiología; entre otras, se plantearon interrogantes centrados en el sujeto que aprende a escribir y leer. $(25,26)$. Estos interrogantes contemplaban cuestiones como: ¿Qué destrezas, habilidades o capacidades requiere cualquier individuo para aprender a leer y escribir?, ¿qué hace el individuo al leer y al escribir?; ¿Qué procesos mentales intervienen para aprender a leer y escribir?; ¿Qué mecanismos se desarrollan a medida que se aprende a leer y escribir?; ¿Qué funciones lingüísticas se alteran cuando la persona no aprende a leer y escribir o tiene dificultades para este aprendizaje?

Las respuestas a estos interrogantes han empezado a configurarse dentro de modelos de la lectura y la escritura acordes con una perspectiva teórica particular, dentro de los que se destacan: i) la perspectiva psicogenética cuyas aplicaciones a la lectura y la escritura tienen como principal exponente a Emilia Ferreiro $(2,26)$; ii) la perspectiva psicolingüística y la perspectiva de la psicología cognitiva, que han descrito los mecanismos psicológicos que permiten la codificación, la decodificación, la composición y la comprensión de un texto. A esta perspectiva corresponde los trabajos desarrollados por Cuetos (23); y iv) la perspectiva socio-constructivista que hace énfasis en el papel del contexto y de la interacción comunicativa en el desarrollo de la lectura y la escritura, y que alcanza su mayor desarrollo en el concepto de alfabetismo emergente $(3,27)$.

La perspectiva psicogenética considera que el niño dispone de una capacidad temprana para escribir y leer. También considera que debido al contexto alfabetizado en que está, éste elabora ideas propias sobre la lectura y la escritura, y formula diversas hipótesis sobre las mismas. Por lo tanto cuando el niño accede a procesos formales de enseñanza de la lectura y la escritura ya posee una serie de conocimientos previos sobre la lengua escrita. (24). El principal impacto de esta postura se dio en la didáctica de la lengua escrita, así como en la comprensión del aprendizaje de la misma en contextos educativos (25).

A partir de la década de los 70's surge la perspectiva psicolingüística, fundamentada en los postulados de la psicología genética y la lingüística generativista que busca describir cuales son los procesos mentales subyacentes al procesamiento de la información escrita, tanto en el momento en que el individuo se encuentra en el proceso de aprendizaje de la misma, como cuando es lector competente (24).

El enfoque cognitivo, también llamado psicología de la lectura y la escritura, concibe la lectura y la escritura como actividades cognitivas complejas que requieren la interacción de una serie de operaciones y subcomponentes que involucran aspectos viso-perceptivos, lingüísticos y cognitivos (4) en este sentido se preocupa principalmente por responder a las preguntas ¿Qué destrezas, habilidades o capacidades requiere cualquier individuo para aprender a leer y escribir?, ¿qué hace el individuo al leer y al escribir?; y ¿Qué procesos mentales intervienen en este aprendizaje?
Actualmente, tanto la perspectiva cognitiva y la psicolingüística se enfocan en el estudio de la manera como el individuo procesa la lengua escrita. Siendo el énfasis de la primera, el estudio del desarrollo de la lengua escrita desde los primeros años hasta la consolidación de estos aprendizajes (28). Mientras que, la perspectiva psicolingüística se ocupa principalmente del procesamiento fonológico y léxico, una vez que las personas han aprendido a decodificar y codificar.

De acuerdo con Flórez y cols (3), el alfabetismo emergente es un concepto que se centra en la socialización temprana del alfabetismo. Se refiere a los conocimientos que adquieren los niños y las niñas respecto a la función, la forma y el contenido de la lengua escrita, así como la actitud que tienen hacia ella antes de la enseñanza formal de la misma, por efecto de la interacción con el material impreso en contextos informales y formales como el hogar y el jardín escolar. Para Bravo Valdivieso (29) el alfabetismo emergente es un modelo cognitivo que explica la evolución del proceso psicológico que se inicia en la toma de conciencia del lenguaje hasta el dominio de la decodificación, la codificación y el reconocimiento visual de las palabras (29). Los estudios sobre el desarrollo de la lectura y la escritura han evidenciado que estos procesos no emergen repentinamente, como parte de un proceso de apropiación cultural en el contexto escolar sino que requieren el desarrollo previo de habilidades cognoscitivas y lingüísticas que sustenten este aprendizaje (3), así como de un entorno que le provea al niño oportunidades para emplear material impreso.

De acuerdo a lo anterior se sabe que existen escenarios y prácticas favorecedoras de la emergencia del alfabetismo, las cuales se fundamentan en dos aspectos: las prácticas evolutivamente apropiadas y las condiciones intra-individuales. Las primeras se centran en las dinámicas de interacción con padres y adultos significativos en torno a actividades relacionados con la lectura y la escritura (lectura compartida, conocimiento de las letras y compromiso de la familia, entre otras); mientras que las segundas involucran actividades asociadas con el desarrollo lingüístico y cognitivo del niño (conciencia fonológica y aprendizaje de vocabulario nuevo) (29).

Para efectos de este estudio la lengua escrita se asume desde las perspectivas cognitiva y psicolingüística, en tanto que su aprendizaje supone el desarrollo de habilidades previas las cuales se instauran antes del aprendizaje formal de la misma; y desde la psicología genética para identificar la etapa del desarrollo de la lectura y escritura en la que se ubican los niños observados. Esta decisión es posible debido a que como se mencionó anteriormente, estas perspectivas comparten postulados sobre el desarrollo de estos procesos (28).

Para poder caracterizar los prerrecurrentes de la lectura y la escritura de un grupo de niños es necesario conocer el desarrollo de estos procesos en condiciones típicas. Con este fin, a continuación se presentan los conceptos fundamentales que guían este estudio.

\section{Desarrollo de la escritura y la lectura}

El desarrollo de la lectura y la escritura ocurre durante los primeros años de vida e involucra tanto las dinámicas de los ambientes naturales como los años preescolares y escolares iniciales. Este desarrollo se da a través de una serie de fases o etapas (26). Para Ferreiro 
(26), la primera Fase de "Pre lectura y preescritura" comprende el período que va desde el nacimiento hasta los 6 años aproximadamente. Durante estos primeros seis años el niño desarrolla la lengua oral, adquiere una serie de conocimientos sobre el mundo que lo rodea, reconoce que el propósito de la lengua escrita es la comunicación y desarrolla habilidades visuales, visomotoras, perceptivo-auditivas y lingüísticas necesarias para iniciar el aprendizaje formal de la lectura y la escritura. Cabe resaltar que debido al carácter dinámico del desarrollo un niño puede superar esta etapa antes de los seis años, pero en ningún caso se espera que permanezca en ella pasado este tiempo $(24,26)$. La evidencia de la forma como el niño adquiere estos prerrecurrentes se observa en el desarrollo de actividades asociadas a las siguientes dimensiones de la decodificación, codificación y comprensión de la lengua escrita.

1. La conciencia fonológica, definida como la habilidad para analizar y sintetizar los segmentos sonoros de la lengua independientemente de su significado. Esta habilidad es considerada como predictora del aprendizaje lector e implica actividades a nivel silábico e intrasilábico como: segmentar palabras en silabas o sonidos, omitir sonidos iniciales o finales de las palabras, o, contar los sonidos que conforman una palabra. $(3,30)$

2. El conocimiento de lo impreso, que corresponde al conocimiento que poseen los niños sobre el material escrito presente en su entorno cotidiano. Incluye: i) el conocimiento del libro como objeto; ii) el conocimiento de lo escrito, es decir, el conocimiento del niño sobre la escritura como sistema. El niño reconoce que las grafías tienen y transmiten significado, reconoce la direccionalidad de la escritura y conoce el aspecto visual de un texto, y iii) el conocimiento de las letras es decir identifica las "grafias", así como el nombre o el fonema que representan algunas letras. (3, 31)

3. El desarrollo de la lengua oral, puesto que al considerar la lectura y la escritura como un continuo dentro del desarrollo del lenguaje, se supone una relación directa entre las habilidades lingüísticas y la emergencia del alfabetismo (32). Además, el desarrollo del lenguaje implica un conjunto de habilidades de comprensión y expresión que se aplican tanto a la oralidad como la escritura (4).

4. Motivación/Interés para aprender, que se constituye un aspecto común para cualquier aprendizaje debido a que el vinculo afectivo/emocional que se crea entre los otros y el niño, entre los niños y los objetos materiales y simbólicos que hacen parte de su cultura así como los acontecimientos y eventos valorados por dicha cultura, promoverán en el niño el deseo de leer y escribir. Desde los contextos psicológico y pedagógico se insiste en la relevancia entre el afecto y la cognición, puesto que los factores afectivo-motivacionales influyen en el desarrollo y aprendizaje de la lectura y la escritura $(3,4)$.

Existen diversas investigaciones que evidencian el impacto de las interacciones cotidianas para el desarrollo de la lectura y la escritura $(3,31)$. En la experiencia diaria, desde edades muy tempranas los niños tienen contacto con la lengua oral y escrita. Observan a los adultos leer o escribir; perciben escrituras en la calle, ven a sus familiares estudiar, todas esas son oportunidades en que los niños empiezan a tratar de entender qué son y para qué son esas formas que no son dibujos (26).

5. Coordinación visomotora, es la habilidad para realizar movimientos que implican precisión, puesto que se coordinan las habilidades visuales con la coordinación de segmentos corporales. Implica actividades como: uso de pinza, prensión, enhebrado, dibujos, collage, coloreado, uso del punzón, construcciones, recortes, y escribir conservando el renglón (33).

El desarrollo de los prerrecurrentes descritos anteriormente sólo es posible gracias a una serie de condiciones biológicas, psicológicas, sociales, ambientales y culturales básicas. Cualquier aprendizaje requiere un soporte biológico, es decir, el niño debe estar en condiciones de salud adecuadas a nivel general y recibir atención oportuna preventiva o asistencial cuando lo requiera. También es necesario el equilibrio socio afectivo, es decir, el niño necesita un ambiente familiar y social apropiado, en el que sea reconocido como individuo y en el que pueda interactuar con otros para establecer relaciones afectivas, normas socioculturales y una serie de aprendizajes incidentales de diversos aspectos que son cruciales para los aprendizajes formales en los contextos educativos $(3,27)$.

Es importante resaltar que la guía del adulto es determinante en esta etapa del desarrollo del niño puesto que mediante la interacción con los adultos y otras personas así como con los eventos propios de su cultura, el niño interioriza funciones cognoscitivas, lingüísticas, sociales y afectivas. Adicional a las condiciones mencionadas antes, el desarrollo de los prerrecurrentes para la lectura y la escritura, así como de cualquier otra destreza o capacidad, implica unas condiciones ambientales básicas entre las que se destacan: vivienda apropiada, acceso a los servicios sanitarios básicos, sustento económico y acceso a contextos de educación preescolar (34).

Cabe aclarar que estos son los prerrecurrentes generales descritos en la literatura para el desarrollo de la lectura y la escritura pero dependiendo del enfoque que se toma algunos ganan mayor relevancia que otros. Para efectos de este estudio, por ejemplo, se dará prelación a la conciencia fonológica, el conocimiento de lo impreso, el razonamiento verbal y el factor motivacional, los cuales se medirán a través del instrumento propuesto. Los aspectos viso-motores y del lenguaje sólo serán monitoreados a partir de la verificación del estado general de desarrollo de los niños participantes para identificar factores de riesgo en el desarrollo de los prerrecurrentes.

\section{Etapas de desarrollo de la escritura}

A partir de las investigaciones de Emilia Ferreiro $(2,26)$ se establecieron las siguientes etapas en el desarrollo de la escritura:

\section{Primer período: \\ Diferenciación Icónica- No icónica}

En este estadio, la demanda cognitiva inicial es diferenciar la lengua escrita del dibujo. El niño en este momento de su desarrollo ya ha asumido como modalidad representativa el dibujo, tiene habilidad para expresarse a través de dibujos. Cuando el niño logra 
diferenciar entre dibujos y los materiales escritos permitirá que la escritura se configure como su nueva modalidad representativa desplazando al dibujo. Es decir, el niño reconoce que a través de una serie de grafías puede generar diversos significados sin depender de los dibujos. Durante esta primera etapa el niño incluye grafías o símbolos similares a las letras dentro de los dibujos que representa y poco a poco va separando la escritura del dibujo conforme asimila que las grafías por si mismas representan significados que no dependen de una imagen.

\section{Segundo periodo:}

diferenciación sobre los ejes cuantitativos y cualitativos

Durante este periodo el niño se centra en elaborar las condiciones de interpretabilidad de sus propios escritos y los demás escritos. Estas condiciones se conocen como "Condiciones formales de los textos". De igual modo, desarrolla principios alrededor de las formas escritas, como el principio cuantitativo, el cual refiere la cantidad mínima de grafías necesarias para escribir o leer un texto. En algunas ocasiones los niños también fijan el máximo de grafías requeridas para cada texto. En esta etapa el niño considera que a través de la escritura puede representar características del elemento al que se refiere, por ejemplo, el tamaño, la edad o su altura, por lo que realiza grafías más grandes o más pequeñas conforme al tamaño del objeto, o emplea grafías mas "altas" si debe escribir el nombre de su papá o mamá porque son mas grandes. Estas ideas del niño hacen parte de la hipótesis de cuantificación del objeto. A lo largo de esta etapa el niño empieza a entender que la forma de las palabras no esta determinada por el objeto al que hacen referencia; el niño también reconoce que las palabras se componen de letras de diversas formas que él trata de imitar en sus trazos y conforma la hipótesis interfigural, es decir el niño asume que la escritura de dos objetos diferentes es diferente. Durante esta etapa el niño mezcla sus propias grafías con las grafías convencionales de su lengua.

\section{Tercer periodo: \\ la fonetización de la escritura}

En este momento el niño reconoce partes internas de las palabras-sílabas- lo que le permite establecer asociaciones sonoras entre las mismas. Durante este periodo el niño logra reconocer la relación grafía-fonema; descubre que a cada una de las unidades mínimas de su expresión oral le corresponde una forma escrita. Sin embargo, esto no implica que el niño establezca de forma inmediata el valor sonoro convencional o su representación grafémica, sino que el niño es consciente de la correlación entre las formas sonoras y las representaciones grafémicas. En esta etapa el niño realiza las grafías convencionales de su lengua.

El periodo de fonetización se divide en tres etapas, cada una de las cuales se caracteriza por una hipótesis específica que refleja el modo en que el niño entiende qué representan las formas escritas. Estas hipótesis de acuerdo a su orden de aparición son:

- Hipótesis silábica: se caracteriza porque el niño fija nuevas condiciones para la representación escrita y su interpretación que le permite regular la cantidad de grafías requeridas para cada escritura aunque eso no implica que empleen las letras adecuadas. La elaboración de esta hipótesis es de gran relevancia para el niño puesto que implica un logro cognitivo que reorganiza todo el sistema de conocimientos sobre la escritura que ha configurado hasta el momento.

Hipótesis silábico-alfabética: en esta etapa el niño empieza a identificar que hay silabas que pueden conformarse por un solo fonema y silabas que tienen dos o más fonemas. Esta etapa constituye la transición entre la escritura propia que el niño ha venido usando y la escritura con las grafías convencionales de la lengua. Se denomina silábico-alfabética porque en un primer momento el niño sólo puede pensar la escritura por sílabas hasta que logra determinar la estructura fonémica de las palabras, lo que lo llevaría al momento alfabética.

Hipótesis alfabética: se presenta cuando el niño comprende la organización del sistema alfabético y empieza a automatizar la escritura de las palabras que son de uso frecuente. Esta etapa coincide con el inicio del primer grado de escolaridad básica.

\section{Etapas de desarrollo de la lectura}

En cuanto al desarrollo de la lectura Ehri (35) plantea las siguientes fases o etapas:

\section{Fase prealfabética}

Constituye la primera fase. Se caracteriza porque los niños comienzan a reconocer las palabras escritas, por medio de algunas características gráficas de las letras, en particular las letras iniciales o finales de las palabras, y a partir de estos rasgos intentan pronunciarlas y adivinar su significado. Esta fase implica que los niños han adquirido conciencia de lo impreso, así como de la representabilidad del significado de las grafías.

\section{Fase alfabética parcial}

En esta fase el reconocimiento de las palabras se basa en una mayor cantidad de signos o letras para lo que es necesaria la participación del procesamiento fonológico de las letras y las sílabas. Esta fase puede desarrollarse antes de la escolaridad formal en la que se inicia la enseñanza de la lectura, sin embargo para su aparición previa al aprendizaje formal, requiere la mediación constante del adulto.

\section{Fase alfabética completa}

En la cual el niño puede reconocer palabras enteras aunque no sea capaz de deletrearlas de forma correcta, por lo general aparece durante el proceso de enseñanza formal de la lectura.

\section{Consolidación alfabética}

El desarrollo de la lectura en el componente de decodificación culmina con esta fase, en la cual el niño aprende a reconocer y decodificar las palabras de poco uso así como las seudopalabras. Esta última fase sólo es posible gracias al procesamiento fonológico.

Es importante señalar que las fases o etapas en el desarrollo de la lectura han sido más complejas de determinar, a diferencia de lo 
que ocurre con las etapas del desarrollo de la lectura, dada la dificultad para evidenciar la manera como se realiza el procesamiento de información o decodificación. Además, el paso de una fase a otra no siempre se puede delimitar de forma precisa, por lo que se sugiere el concepto de continuidad entre ellas.

\section{METODOLOGÍA}

El presente estudio es de tipo descriptivo en tanto que se ocupó de diseñar una prueba tamiz de prerrecurrentes de lectura y escritura en población preescolar. El método adoptado fue el propuesto por Cohen \& Swerdlik (37), para la elaboración de pruebas. La investigación se llevó a cabo en cinco etapas: conceptualización de la prueba; construcción de la prueba; ensayo de la prueba, análisis de reactivos y revisión de la prueba.

Para la primera etapa se hizo una consulta a profundidad de los principales autores y exponentes de las teorías que explican y describen el proceso de aprendizaje y desarrollo de la lectura y la escritura. Esta búsqueda permitió identificar la existencia de cuatro cuerpos teóricos que abordan esta temática: la teoría psicolingüística, la psicología genética, la psicología cognitiva y la teoría socioconstructivista. Luego de este análisis se procedió a identificar las convergencias y divergencias existentes entre tales cuerpos teóricos en torno a lo que en este estudio se ha decidido denominar "prerrecurrentes" (1-4), (22-31,35). De la misma manera se consultó el contenido y estructura de un grupo de pruebas reconocidas por su eficacia y pertinencia en la evaluación del lenguaje, desde estas mismas perspectivas teóricas (5-11).

Para la fase de construcción de la prueba se identificaron los constructos a evaluar con sus respectivas dimensiones y subdimensiones. Posteriormente a cada subdimensión se le asignó un grupo de indicadores con sus respectivos índices para la prueba. El criterio para la incorporación de los indicadores, así como para la selección de los índices se soportó en los resultados del análisis que se hizo a las pruebas existentes, en confrontación con los hallazgos teóricos. La validez de esta primera fase de diseño se alcanzó a través de la consulta a 8 jueces expertos, quienes evaluaron la pertinencia y suficiencia del instrumento. De esta primera validación se derivaron algunos ajustes que fue preciso incorporar al instrumento antes de su prueba piloto. Los ajustes sugeridos involucraban aspectos tanto de contenido como de forma de la prueba.

Finalmente, el ensayo de la prueba se llevó a cabo a través de un análisis cualitativo de los reactivos (37), basado en los resultados de la aplicación de la prueba a un grupo de 50 niños y niñas preescolares que se encontraban entre los 2 y los 6 años de edad. Para el efecto, antes de la aplicación de la prueba se solicitó el consentimiento informado al representante legal de cada niño y la aprobación de la institución educativa en la cual se llevó a cabo la aplicación del instrumento. La prueba fue administrada por 3 estudiantes de último semestre de fonoaudiología previamente entrenados en la aplicación de la misma y con amplio conocimiento sobre los procesos normales de desarrollo del lenguaje en niños. Todas las aplicaciones fueron supervisadas por la investigadora principal del estudio.

El análisis cualitativo de la prueba se enfocó en los 13 aspectos recomendados por Cohen \& Swerdlik (37) para tal fin, que tienen que ver con la recuperación de las percepciones de los aplicadores de la prueba sobre la calidad de la misma. El registro de estos análisis se hizo de forma escrita a través de las respuestas que dieron los aplicadores a preguntas relacionadas con el ambiente de la prueba, la imparcialidad de la misma, el lenguaje que emplea y la longitud.

Dado que la prueba piloto fue aplicada a un grupo de niños cuyo desarrollo se considera dentro de los rangos de normalidad esperados para su edad cronológica, esta prueba piloto también se uso como referencia para evaluar la sensibilidad del instrumento al desarrollo evolutivo de los prerrecurrentes de lectura y escritura. Luego de este proceso se realizaron los ajustes pertinentes y se produjo la versión definitiva de la misma.

\section{RESULTADOS}

El diseño de la prueba tamiz de prerrecurrentes de lectura y escritura para población preescolar se llevó a cabo siguiendo los pasos sugeridos por Cohen \& Swerdlik (40), quienes proponen que la elaboración de una prueba contempla cinco etapas: conceptualización de la prueba, construcción de la prueba, ensayo de la prueba, análisis de reactivos y revisión de la prueba. A continuación se describe el proceso de desarrollo de cada uno de estas etapas, así como los hallazgos de las mismas.

Para la etapa de conceptualización de la prueba se consultaron 38 fuentes bibliográficas y 17 baterías de evaluación relacionadas con el tema de aprendizaje de la lectura, la escritura y el desarrollo del lenguaje. De esta primera fase se concluyó que existen cuatro campos de estudio transdisciplinares que abordan este objeto de estudio: la psicolingüística, la psicología genética, la psicología cognitiva y la socio-constructivista. Además se identificaron las divergencias y convergencias de estos campos en cuanto al abordaje y explicación de cómo se produce el aprendizaje de la lectura y la escritura, qué destrezas, habilidades y capacidades requiere un individuo para aprender a leer y escribir, qué mecanismos cognitivos, lingüísticos, motores, sensoperceptivos y afectivos se desarrollan a medida que se avanza en este aprendizaje, y qué funciones lingüísticas se alteran cuando la persona presenta dificultades para alcanzar estos desempeños.

Sobre la base de estos hallazgos, en la fase de construcción de la prueba se identificaron 7 dimensiones que componen el constructo prerrecurrentes de la lectura y la escritura, las cuales se describen a continuación

- La conciencia fonológica, cuya presencia puede monitorearse a través de la habilidad que demuestre el niño evaluado para segmentar palabras en silabas o sonidos, omitir sonidos iniciales o finales de las palabras y contar los sonidos que la conforman.

El conocimiento de lo impreso, que puede evaluarse a través del conocimiento que demuestre el niño sobre el material escrito. Es decir, su capacidad para reconocer que los libros, las letras y el material impreso en general se puede leer. Corresponde a acciones que ejecuta el niño tales como asignar significados y sentido a los contenidos escritos, aún cuando no sabe leer. 


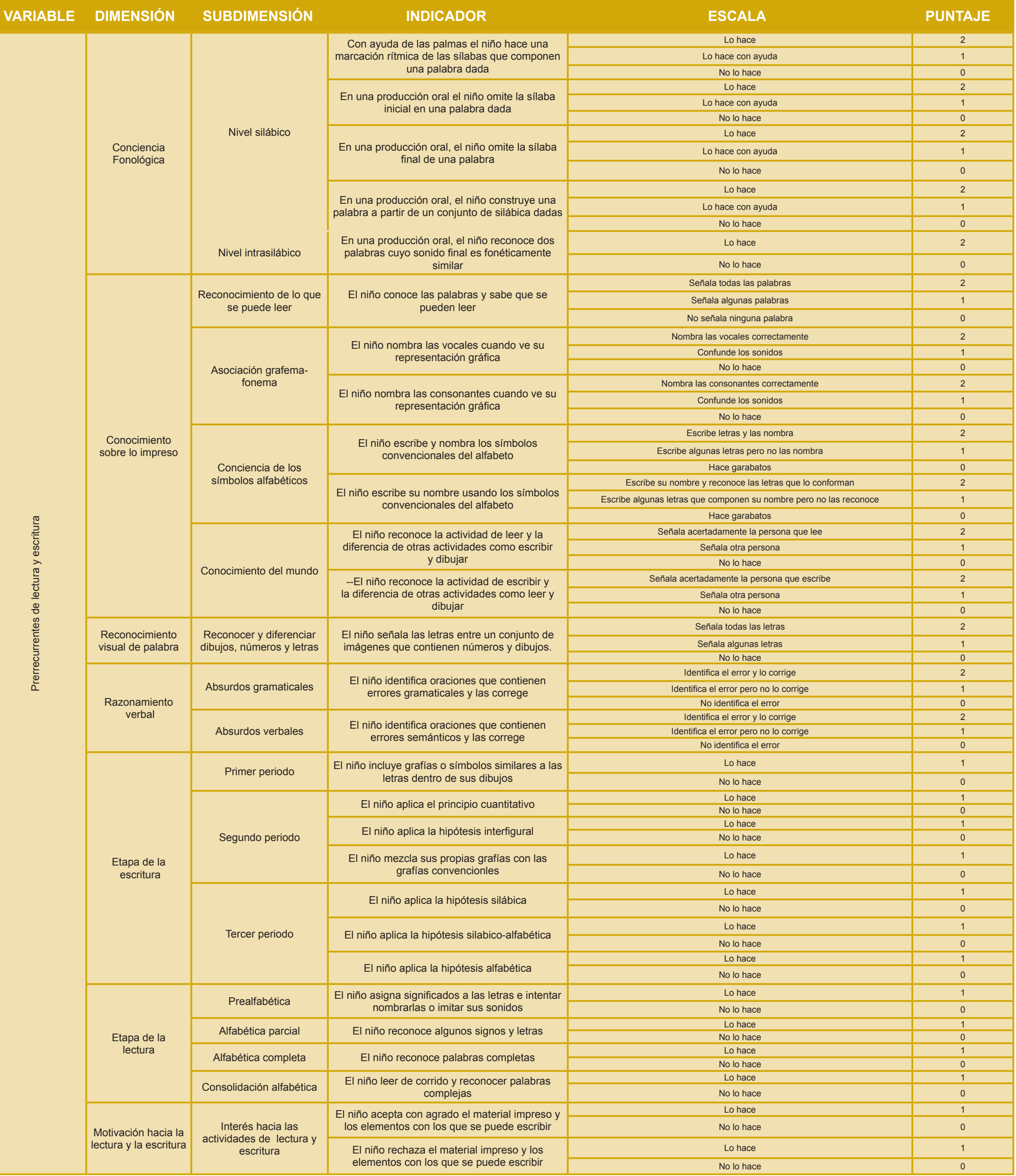

Tabla 1. Dimensiones e indicadores del constructo a medir

REV. COL. REH || Bogotá, Colombia || Volumen 10 || Página 46 - 58 || Noviembre 2011 || ISSN 1692 - 1879 
El reconocimiento visual de la palabra, cuya presencia puede monitorearse a través de la habilidad que demuestre el niño evaluado para reconocer y diferenciar los dibujos de las letras, y las letras de los números.

- El razonamiento verbal, que se evalúa observando la habilidad que demuestra el niño para valorar la coherencia de la estructura y el sentido del lenguaje oral. Es decir, para identificar la presencia de absurdos verbales en las oraciones, tanto de tipo semántico como de tipo sintáctico.

- La motivación para leer y escribir, que se observa en la actitud que demuestra el niño cuando se le solicita que ejecute tareas de lectura y escritura, aun cuando no sabe leer ni escribir.

- Etapa de la lectura, corresponde al grado de aproximación que logra el niño hacia la decodificación completa del texto escrito.

- Etapa de la escritura, comprende el grado de aproximación que logra el niño hacia la codificación completa del texto escrito

Posteriormente, y atendiendo a los hallazgos identificados en las otras pruebas revisadas, se identificaron las subdimensiones con sus respectivos indicadores, y de ellos se derivaron los ííndices a incluir en la prueba. La tabla 1., muestra la manera como se hizo dicha asignación.

Con el propósito de alcanzar un mayor nivel de validez para la prueba, luego de esta primera fase se procedió a consultar a 8 jueces expertos en cuanto a la pertinencia y la suficiencia de los ítems. De acuerdo con esta valoración los jueces sugirieron diferenciar las tareas asociándolas a lo rangos etéreos de los niños a evaluar. Así mismo, sugirieron algunos cambios en la organización de los items dentro de la prueba; en la forma en que se debían dar algunas instrucciones a los niños; $y$, en el criterio de calificación de los desempeños (índices). Adicionalmente, se sugirió dar paso al proceso de pilotaje de prueba.

A continuación se describen los principales cambios hechos a la prueba por sugerencia de los expertos consultados:

- Incorporación de las dos pruebas ( 2 a 3 años y 3 a 5 años) en una sola en la que se diferencie cada tarea según el grupo etareo al cual se le aplicará.

- Separación del instructivo para el aplicador ,de la hoja de respuestas para el evaluador, y de los materiales visuales de la misma.

- Privilegiar las fotografías en las ayudas visuales dado que son de más fácil reconocimiento que los dibujos.

Luego de este proceso de validación se realizaron los ajustes sugeridos para lo cual: se eligieron los ítems para cada indicador haciendo un diferencial entre las que se consideran evolutivamente apropiadas para dos rangos de edades: entre los 2 años y los 2 años 11 meses, $y$ entre los 3 años y los 5 años 11 meses. Este diferencial se considero necesario para los ítems de segmentación silábica, omisión de sílaba inicial y sílaba final, síntesis silábica, asociación grafema-fonema, reconocimiento visual de palabra y conciencia de los símbolos alfabéticos. Así mismo se cambiaron las instrucciones de algunas tareas y el orden de aparición de otras. Como resultado de este mismo proceso, se decidió separar el instructivo de aplicación de la prueba de la hoja de calificación; así como unificar el proceso de calificación de los desempeños.

Para la fase de ensayo de la prueba y análisis de los reactivos se llevó a cabo una prueba piloto con 50 niños con edades comprendidas entre los 2 y los 6 años, y con niveles de desarrollo considerados dentro de los rangos de normalidad. A continuación se describen los desempeños obtenidos por los participantes en la prueba piloto.

En la prueba piloto participaron 26 niñas y 24 niños pertenecientes a los cursos de prejardín, jardín y transición de un jardín infantil de la ciudad de Bogotá. La prueba fue aplicada de forma individual a cada niño con una duración de aproximadamente 20 minutos por participante. La evaluación se hizo dentro de la misma institución educativa, a lo largo de la jornada escolar.

La tabla 2, muestra la distribución de los participantes por género y la tabla 3, indica la distribución de la población evaluado por grupo etareo.

\begin{tabular}{|c|c|}
\hline GÉNERO & PARTICIPANTES \\
\hline Femenino & 26 \\
\hline Masculino & 24 \\
\hline
\end{tabular}

Tabla 2. Distribución de la población por género

\begin{tabular}{|c|c|}
\hline EDAD & PARTICIPANTES \\
\hline 2 a 2.11 años & 4 \\
\hline 3 a 3.11 años & 15 \\
\hline 4 a 4.11 años & 16 \\
\hline 5 a 5.11 años & 12 \\
\hline 6 a 6.11 años & 3 \\
\hline
\end{tabular}

Tabla 3. Distribución de la población por rango etareo

En cuanto a los desempeños de los participantes en las subpruebas, la tabla 4, muestra los resultados obtenidos en la dimensión: conciencia fonológica.

Como se observa en la tabla 4., en su mayoría los niños de 2 a 2,11 años logran desarrollar las tareas de segmentación de palabras de dos sílabas, pero presentan dificultades para segmentar las palabras de tres sílabas. Así mismo, los niños de 3 años en adelante logran segmentar tanto las palabras de dos sílabas como las de tres, lo cual demuestra que el margen de error en esta tarea disminuye a medida que aumenta la edad.

Los niños de 3 y 4 años presentan dificultades para omitir la sílaba inicial tanto en palabras bisílabas como trisílabas. Al igual que 
en la tarea anterior, la probabilidad de ejecutar acertadamente esta actividad se incrementa con la edad, motivo por el cual los niños de 5 años muestran un mejor desempeño y todos los de 6 años logran ejecutarla.

\begin{tabular}{|c|c|c|c|c|}
\hline \multirow{2}{*}{ TAREA } & \multirow{2}{*}{ EDAD } & \multicolumn{3}{|c|}{ DESEMPEÑOS } \\
\hline & & NO LO HACE & CON AYUDA & LO HACE \\
\hline \multirow{5}{*}{$\begin{array}{l}\text { Reconocimiento de } \\
\text { lo que se puede leer }\end{array}$} & 2-2,1 años & 3 & 0 & 1 \\
\hline & 3-3,11 años & 5 & 5 & 5 \\
\hline & 4-4,11 años & 2 & 2 & 11 \\
\hline & 5-5,11 años & 0 & 0 & 12 \\
\hline & 6 años & 0 & 0 & 3 \\
\hline \multirow{5}{*}{$\begin{array}{l}\text { Asociación grafema- } \\
\text { fonema }\end{array}$} & 2-2,1 años & 1 & 3 & 0 \\
\hline & 3-3,11 años & 18 & 5 & 1 \\
\hline & 4-4,11 años & 13 & 4 & 14 \\
\hline & 5-5,11 años & 1 & 2 & 20 \\
\hline & 6 años & 0 & 0 & 6 \\
\hline $\begin{array}{l}\text { Reconocimiento de } \\
\text { dibujos y letras }\end{array}$ & 2-2,1 años & 0 & 3 & 0 \\
\hline \multirow{5}{*}{$\begin{array}{l}\text { Conocimiento del } \\
\text { mundo }\end{array}$} & 2-2,1 años & 0 & 3 & 5 \\
\hline & 3-3,11 años & 3 & 10 & 17 \\
\hline & 4-4,11 años & 0 & 1 & 23 \\
\hline & 5-5,11 años & 0 & 1 & 23 \\
\hline & 6 años & 0 & 0 & 6 \\
\hline
\end{tabular}

Tabla 4. Desempeños de los participantes en las tareas de conciencia fonológica.

Manteniendo la misma tendencia, se evidencia una dificultad general de los niños de 4 años para omitir la sílaba final de palabras tanto bisílabas como trisílabas. Este desempeño mejora a medida que se incrementa la edad, lo cual se evidencia en el desempeño logrado por los niños de 6 años quienes ejecutan la tarea sin ninguna dificultad.

Se evidencia una tendencia a ejecutar acertadamente las tareas de síntesis silábica en todos los rangos etareos ( 4 a 6 años). El margen de error en esta tarea también disminuye a medida que incrementa la edad de los niños evaluados.

Los niños de 2 y 3 años presentan dificultades para ejecutar la tarea de identificación de sonidos semejantes al final de la palabra. El desempeño en la ejecución de esta tarea también mejora a medida que se incrementa la edad.

En cuanto al conocimiento de lo impreso, la tabla 5., permite ver los desempeños alcanzados por la población evaluada.

Como se observa, los niños de 2 años muestran dificultades para reconocer lo que se puede leer. $\mathrm{Al}$ igual que en las otras tareas, este desempeño mejora a medida que se incrementa la edad de los niños evaluados.

La asociación grafema-fonema en la lectura se evidencia en los niños de 4 años en adelante y en igual proporción tanto para las vocales como para las consonantes. La correspondencia entre fonema y grafema en la escritura sólo se identificó en los niños de 4 años en adelante.

\begin{tabular}{|c|c|c|c|c|}
\hline \multirow{2}{*}{ TAREA } & \multirow{2}{*}{ EDAD } & \multicolumn{3}{|c|}{ DESEMPEÑOS } \\
\hline & & NO LO HACE & CON AYUDA & LO HACE \\
\hline \multirow{5}{*}{$\begin{array}{l}\text { Reconocimiento } \\
\text { de lo que se } \\
\text { puede leer }\end{array}$} & 2-2,1 años & 3 & 0 & 1 \\
\hline & 3-3,11 años & 5 & 5 & 5 \\
\hline & 4-4,11 años & 2 & 2 & 11 \\
\hline & 5-5,11 años & 0 & 0 & 12 \\
\hline & 6 años & 0 & 0 & 3 \\
\hline \multirow{5}{*}{$\begin{array}{c}\text { Asociación } \\
\text { grafema-fonema }\end{array}$} & 2-2,1 años & 1 & 3 & 0 \\
\hline & 3-3,11 años & 18 & 5 & 1 \\
\hline & 4-4,11 años & 13 & 4 & 14 \\
\hline & 5-5,11 años & 1 & 2 & 20 \\
\hline & 6 años & 0 & 0 & 6 \\
\hline $\begin{array}{l}\text { Reconocimiento } \\
\text { de dibujos y } \\
\text { letras }\end{array}$ & 2-2,1 años & 0 & 3 & 0 \\
\hline \multirow{5}{*}{$\begin{array}{l}\text { Conocimiento del } \\
\text { mundo }\end{array}$} & 2-2,1 años & 0 & 3 & 5 \\
\hline & 3-3,11 años & 3 & 10 & 17 \\
\hline & 4-4,11 años & 0 & 1 & 23 \\
\hline & 5-5,11 años & 0 & 1 & 23 \\
\hline & 6 años & 0 & 0 & 6 \\
\hline
\end{tabular}

Tabla 5. Desempeños de los participantes en las tareas de conocimiento sobre lo impreso.

Los niños de 2 años diferencias los dibujos de las letras, y en todos los rangos etareos los niños logran diferenciar las tareas de leer y escribir de otras actividades. Esta destreza, al igual que las otras, se hace más evidente en los niños mayores ( 5 y 6 años).

La tabla 6., muestra los desempeños de los participantes en las tareas de razonamiento verbal. Como se observa, la capacidad para identificar absurdos gramaticales sólo se consolida en los grupos etareos de 5 y 6 años. Mientras que en el caso de los absurdos verbales se nota mayor facilidad para identificarlos a partir de los 3 años de edad.

\begin{tabular}{|c|c|c|c|c|}
\hline \multirow{2}{*}{ TAREA } & \multirow{2}{*}{ EDAD } & \multicolumn{3}{|c|}{ DESEMPEÑOS } \\
\hline & & NO LO HACE & CON AYUDA & LO HACE \\
\hline \multirow{5}{*}{$\begin{array}{l}\text { Absurdos } \\
\text { gramaticales }\end{array}$} & 2-2,1 años & 9 & 3 & 0 \\
\hline & 3-3,11 años & 24 & 17 & 4 \\
\hline & 4-4,11 años & 20 & 20 & 5 \\
\hline & 5-5,11 años & 1 & 3 & 32 \\
\hline & 6 años & 0 & 0 & 9 \\
\hline \multirow{5}{*}{$\begin{array}{l}\text { Absurdos } \\
\text { verbales }\end{array}$} & 2-2,1 años & 4 & 0 & 8 \\
\hline & 3-3,11 años & 7 & 4 & 31 \\
\hline & 4-4,11 años & 4 & 1 & 40 \\
\hline & 5-5,11 años & 0 & 1 & 35 \\
\hline & 6 años & 0 & 0 & 9 \\
\hline
\end{tabular}

Tabla 6. Desempeños de los participantes en las tareas de razonamiento verbal.

La tabla 7., muestra la ubicación de los participantes en las etapas de la lectura. 


\begin{tabular}{|l|c|c|c|c|c|c|}
\hline \multicolumn{1}{|c|}{ TAREA } & $\begin{array}{c}2 \text { a } 2 \text { años } \\
11 \text { meses }\end{array}$ & $\begin{array}{c}\text { 3 a } 3 \text { años } \\
11 \text { meses }\end{array}$ & $\begin{array}{c}4 \text { a } 4 \text { años } \\
11 \text { meses }\end{array}$ & $\begin{array}{c}5 \text { a } 5 \text { años } \\
11 \text { meses }\end{array}$ & $\begin{array}{c}6 \text { a } 6 \text { años } \\
11 \text { meses }\end{array}$ \\
\hline Prealfabética & 4 & 14 & 7 & 3 & 0 \\
\hline $\begin{array}{l}\text { Alfabética } \\
\text { parcial }\end{array}$ & 0 & 1 & 5 & 0 & 0 \\
\hline $\begin{array}{l}\text { Alfabética } \\
\text { completa }\end{array}$ & 0 & 0 & 0 & 2 & 1 \\
\hline $\begin{array}{l}\text { Consolidación } \\
\text { alfabética }\end{array}$ & 0 & 0 & 1 & 6 & 2 \\
\hline
\end{tabular}

Tabla 7. Etapa de la lectura por rango etareo.

Al respecto se observa que todos los niños de 2 y 3 años se ubican en la etapa pre-alfabética, mientras que en el grupo etareo de los 4 a los 4,11 años se incrementa el número de niños que se ubican en la etapa alfabética parcial $(41,6 \%)$. Hacia los 5 años en adelante, la mayoría de los niños se ubican en la etapa de consolidación alfabética ( $54,5 \%$ y $66,6 \%$ respectivamente).

De la misma manera la tabla 8, muestra la clasificación de los participantes por etapa de la escritura.

\begin{tabular}{|l|c|c|c|c|}
\multicolumn{1}{|c|}{ TAREA } & $\begin{array}{c}3 \text { a } 3 \text { años } \\
11 \text { meses }\end{array}$ & $\begin{array}{c}4 \text { a } 4 \text { años } \\
11 \text { meses }\end{array}$ & $\begin{array}{c}5 \text { a } 5 \text { años } \\
11 \text { meses }\end{array}$ & $\begin{array}{c}6 \text { a } 6 \text { años } \\
11 \text { meses }\end{array}$ \\
\hline Primer periodo & 13 & 4 & 0 & 0 \\
\hline Segundo periodo & 1 & 8 & 3 & 1 \\
\hline $\begin{array}{l}\text { Tercer periodo } \\
\text { (nombrar la hipótesis) }\end{array}$ & 0 & 2 & 9 & 2 \\
\hline
\end{tabular}

Tabla 8. Etapa de la escritura por rango etáreo.

Respecto a la etapa de la escritura los niños de 3 a 3,11 años se ubican principalmente en el primer periodo, mientras que entre los niños de 4 a 4,11 años se nota una tendencia a ubicarse entre el primer y segundo periodo. Ya en los rangos etareos de 5 y 6 años, en su mayoría los niños se ubican en el tercer periodo.

Finalmente, la tabla 9, muestra el interés que mostraron los niños durante la realización de la prueba hacia las tareas de lectura y escritura.

\begin{tabular}{|c|c|c|c|c|}
\hline \multirow{2}{*}{ TAREA } & \multirow{2}{*}{ EDAD } & \multicolumn{3}{|c|}{ DESEMPEÑOS } \\
\hline & & SIN INTERÉS & POCO INTERÉS & MUCHO INTERÉS \\
\hline \multirow{5}{*}{$\begin{array}{c}\text { Motivación } \\
\text { hacia la } \\
\text { lectura y } \\
\text { escritura }\end{array}$} & 2-2,11 años & 0 & 0 & 3 \\
\hline & 3-3,11 años & 0 & 4 & 11 \\
\hline & $4-4,11$ años & 1 & 4 & 8 \\
\hline & 5-5,11 años & 0 & 0 & 11 \\
\hline & 6 años & 0 & 0 & 3 \\
\hline
\end{tabular}

Tabla 9. Motivación hacia la lectura y la escritura de los participantes.

Como se observa, en todos los rangos etareos se identificaron altos niveles de motivación e interés por las tareas de lectura y escritura, con una mayor tendencia en los rangos de 2 a 2,11 y los 6 años.

De este proceso de pilotaje de la prueba se concluyó que el instrumento es sensible a los cambios evolutivos propios del rango etareo para el que fue diseñado, así como a la presencia de dificultades y alteraciones del desarrollo del lenguaje que afectan los mecanismos cognitivos y lingüísticos necesarios para el adecuado desarrollo de la lectura y la escritura. Se identificaron como fortalezas de la prueba la claridad y usabilidad del instructivo y la hoja de calificación, así como la pertinencia de las ayudas visuales de las tareas que así lo requieren.

Como elementos a mejorar se evidenció la necesidad de ajustar la instrucción para las tareas de segmentación de palabras y omisión de sílabas (inicial y final). Sobre este particular resulta más recomendable usar rimas y juegos rítmicos que faciliten la tarea al niño. En este mismo sentido, se hace necesario ajustar la instrucción para el ítem de asociación grafema-fonema, agregando un tarea distinta a la de escribir el nombre.

\section{DISCUSIÓN}

El aprendizaje y posterior desarrollo de los procesos de lectura y escritura es un campo de estudio transdisciplinar que ha sido abordado por diferentes disciplinas y profesiones tanto desde la perspectiva clínica como desde un enfoque psicopedagógico. Los primeros se han centrado especialmente en el estudio de las alteraciones de este desarrollo, mientras que los segundos se han ocupado especialmente de su enseñanza.

Al día de hoy una de las perspectivas más fuertes en el abordaje de esta temática tiene que ver con el reconocimiento de la importancia de proveer a los niños los escenarios, interacciones y experiencias necesarias para garantizar el apropiado acceso a la lectura y la escritura. Esta mirada amplia del proceso convoca la acción colectiva de profesionales, profesores, padres de familia y cuidadores en la consecución de este objetivo. Lo cual implica que cada actor tenga clara su función dentro del proceso, y para el caso particular de los profesionales especializados, que éstos sepan guiar a los demás, en la ejecución de dicha tarea.

El fonoaudiólogo como profesional experto debe propender por favorecer tanto el acceso de los niños a la lectura y la escritura, como el desarrollo y afianzamiento favorable de este proceso a lo largo de la vida. Para ello, el profesional requiere contar con herramientas especificas que le permitan recoger evidencia de base sobre la cual formular futuros proceso de intervención. Con este propósito se ha diseñado la prueba tamiz de prerrecurrentes de lectura y escritura para población preescolar PTPLE, la cual se espera, se convierta en un apoyo para el ejercicio profesional del fonoaudiólogo en el campo clínico y educativo.

La PTPLE tiene como propósito identificar la ocurrencia de conocimientos, habilidades y destrezas de tipo cognitivo, metacognitivo, lingüístico y actitudinal que se consideran precursores para el aprendizaje y adecuado desarrollo de la lectura y la escritura, y que se hallan presentes en condiciones normales en la primera infancia. Esta prueba no se considera un examen diagnóstico dado que sus resultados sólo dan cuenta de la presencia o ausencia de algunos conocimientos y habilidades asociadas a los desempeños cognitivos, comunicativos y procedimentales involucrados en las tareas de lec- 
tura y escritura. Para su aplicación y uso es necesario tener en cuenta que el desarrollo se evidencia de forma particular en cada niño o niña; que éste, a pesar de ser un continuo, no es necesariamente lineal; y que por ello, en él ocurren saltos, retrocesos y estancamientos que se consideran normales en tanto que preceden a futuros procesos de afianzamiento de las destrezas alcanzadas. Cualquier proceso diagnóstico que se derive de la aplicación del PTPLE debe acompañarse de otros procedimientos cuantitativos y cualitativos de soporte, así como de un adecuado juicio clínico de profesionales expertos en el área. También es importante aclarar que como en cualquier otra prueba, la calidad de los resultados que ésta arroje está directamente relacionado con la habilidad del aplicador para desarrollarla, así como de su nivel de entrenamiento en su uso. De la misma manera, influirán en los resultados los materiales de apoyo que se usen para su aplicación, así como las condiciones ambientales en la que se lleve a cabo, y la disposición de los niños en el momento de su realización. Por tanto, es importante tener en cuenta estos aspectos antes de usarla.
Es importante aclarar que, aunque la prueba piloto demostró la sensibilidad del instrumento a los cambios en el desarrollo que se producen a medida que la edad cronológica avanza, es importante que futuras investigaciones validen esta propiedad del instrumento tanto en población considerada con desarrollo normal, como en poblaciones que presenten deficiencias del desarrollo asociadas al aprendizaje de la lectura y la escritura. Cabe resaltar que dichas investigaciones deben ser rigurosas en la aplicación de criterios de inclusión y exclusión de los participantes sobre este particular.

Así mismo, se recomienda que futuras investigaciones se ocupen de validar el instrumento aquí diseñado de manera que sirva como herramienta esencial para el ejercicio profesional del fonoaudiólogo, para estudiantes en formación y para cualquier otro profesional interesado en el tema. Así mismo, se espera que otros estudios empleen el PTPLE como herramienta de caracterización de los prerrecurentes de lectura y escritura en poblaciones específicas con y sin discapacidad y/o en situación de vulnerabilidad.

\section{Referencia:}

Las referencias a otras obras son una parte muy importante en la literatura científica; ya que estas permiten conocer más sobrę los autores y mantener vivas sus voces dentro del texto.

Flórez R. (Ed.). El lenguaje en la educación. Una perspectiva fonoaudiológica. Bogotá: Universidad Nacional de Colombia; 2003.

2. Ferreiro E. Pasado y presente de los verbos leer y escribir. México: Fondo de Cultura económica; 2001.

3. Flórez R., Restrepo M., Schwanenflugel P. Alfabetismo emergente. Investigación, teoría y práctica. El caso de la lectura. Bogotá: Universidad Nacional de Colombia; 2007.

4. Defior S. Las dificultades de aprendizaje un enfoque cognitivo. Lectura, escritura, matemáticas. Buenos Aires: Ediciones Aljibe; 2003.

5. Puyuelo M., Wiig E., Renom J., Solanos, A. Batería de lenguaje objetiva y criterial B.L.O.C. Barcelona: Editorial Mansson, S.A; 2000.

6. Aguinaga G., López de Suso M.L., fraile A., Olangua P., Uriz, N. Prueba de lenguaje oral de navarra. PLON. Barcelona: Gobierno de Navarra; 1988.

7. Newvorg J., Stock J., Wnek, L. Battelle inventario de desarrollo. Madrid: TEA Ediciones; 1998.
8. Zimmerman. Preschool language scale PLS-3. The corporation harcour brace jovanovich, Inc; 1992.

López M.J., Zurita D., Rendón A., García I., Santamaría M., Iniesta J. ELCE. Buenos Aires: Ciencia de la educación preescolar y especial; 1996.

10. Wernicke C. Test figura palabra - vocabulario expresivo. San Francisco, california: Garner; 1985 .

11. Ortiz M., Jiménez J. Prueba de conocimientos sobre el lenguaje escrito CLE. España: TEA Ediciones; 1993.

12. Kirk S., McCarthy J., Kirk W. Test Illinois de aptitudes psicolingüísticas. Madrid: TEA Ediciones; 1994.

13. Lloyd D. Peabody picture vocabulary test. 1959.

14. Pinasco I., Feldman A., Canepa Z. Escala beta. Buenos Aires: Marymar Ediciones; 1992.

15. Feldman J., Torres M.G. Escala alfa para la evaluación del lenguaje escrito en niños de 3 a 17 años. Buenos Aires: Ediciones Marymar; 1992. 
16. Aguinaga G., López de Suso M.L., fraile A., Olangua P., Uriz N. Prueba de lenguaje oral de navarra. PLON. Barcelona: Gobierno de Navarra; 1988.

17. Aguado G. El desarrollo de la morfosintaxis en el niño de 2 a 7 años TSA. Madrid: Cpe. S.A; 1989.

18. Goldberg G., Van Ert J. Desarrollo auditivo en secuencia lógica. DASL II. Bogotá: CINDA; 1993.

19. Aguilar E., Serra Raventos M. Análisis de la fonética y la fonología infantil. AREHA. Barcelona: Gobierno de Navarra; 2003.

20. Gómez P., Valero J., Buades R., Pérez, A. Test de habilidades metalingüísticas. Madrid: Editorial EOS; (s.f.).

21. Ong W. Oralidad y escritura tecnologías de la palabra. Mexico: Fondo de Cultura Económica; 1987.

22. Yule G. El lenguaje. 3ra edición. Madrid: Ediciones Akal; 2007.

23. Cuetos F. Psicología de la escritura. Madrid: S.A CISSPRAXIS; 2008.

24. Szmigielski M. Psicogénesis de la lengua escrita. El proceso de construcción de la escritura durante la infancia. Buenos Aires: Longseller S.A.; 2002.

25. Tolchinsky L. Aprendizaje del lenguaje escrito. Procesos evolutivos e implicaciones didácticas. México: Editorial Anthropos; 1993.

26. Ferreiro E., Teberosky A. Los sistemas de escritura en el desarrollo del niño. Buenos Aires: Paidos; 1999.

27. Hall N, Larson J, Marsh J. (Edit) Handbook or Early Childhood Literacy. California: SAGE; 2006.

28. Carriillo M, Marin J. Desarrollo metafonológico y adquisición de la lectura: un programa de entrenamiento. Madrid: Centro de investigación y documentación Educativa; 1996.
29. Bravo L. Lectura inicial y Psicología Cognitiva. Santiago: Ediciones universidad católica de chile; 2003.

30. Eslava J, Mejía L. Conciencia fonológica y aprendizaje lector. Bogotá: Acta Neurológica Colombiana. 2008; 24 (S2): 1-29.

31. Andrés M., Urquijo S., Navarro J, García M. Contexto alfabetizador familiar: relaciones con la adquisición de habilidades prelectoras y desempeño lector. European Journal of Education and Psychology. 2010; Vol. 3 (1): 129-140.

32. Guevara Y., López A.; García, G. Habilidades de lectura en el primer grado en alumnos de estrato sociocultural bajo. Revista Mexicana de Investigación. 2008; Vol 13 (37): 573-597.

33. Maruny Ll., Ministral M., Miralles M. Escribir y leer. Materiales curriculares para la enseñanza y el aprendizaje del lenguaje escrito de tres a ocho años. Volúmen I. Madrid: EDELVIVES; 1998.

34. Álvarez, B. Niños campesinos desplazados por la violencia: una nueva minoría cultural en las escuelas urbanas colombianas. Rev Fac Med Univ Nac Colomb. 2006; Vol. 54 (3): 219-224.

35. Ehri L. Phases of development in learning t oread words. En: Oakhill J, Beard R. Reading Development and the teaching of reading. A psychological perspective. Oxford: Blackwell; 1999.

36. Justice L., Invernizzi J. Designing and Implementing an Early Literacy Screening Protocol: Suggestions for the Speech-Language Pathologist. Language, speech and hearing services in schools. 2007; Vol. 33 (2): 84-101.

37. Cohen R., Swerdlik M. Pruebas y evaluación psicológica. introducción a las pruebas y a la medición. México: Mc Graw Hill; 2001.

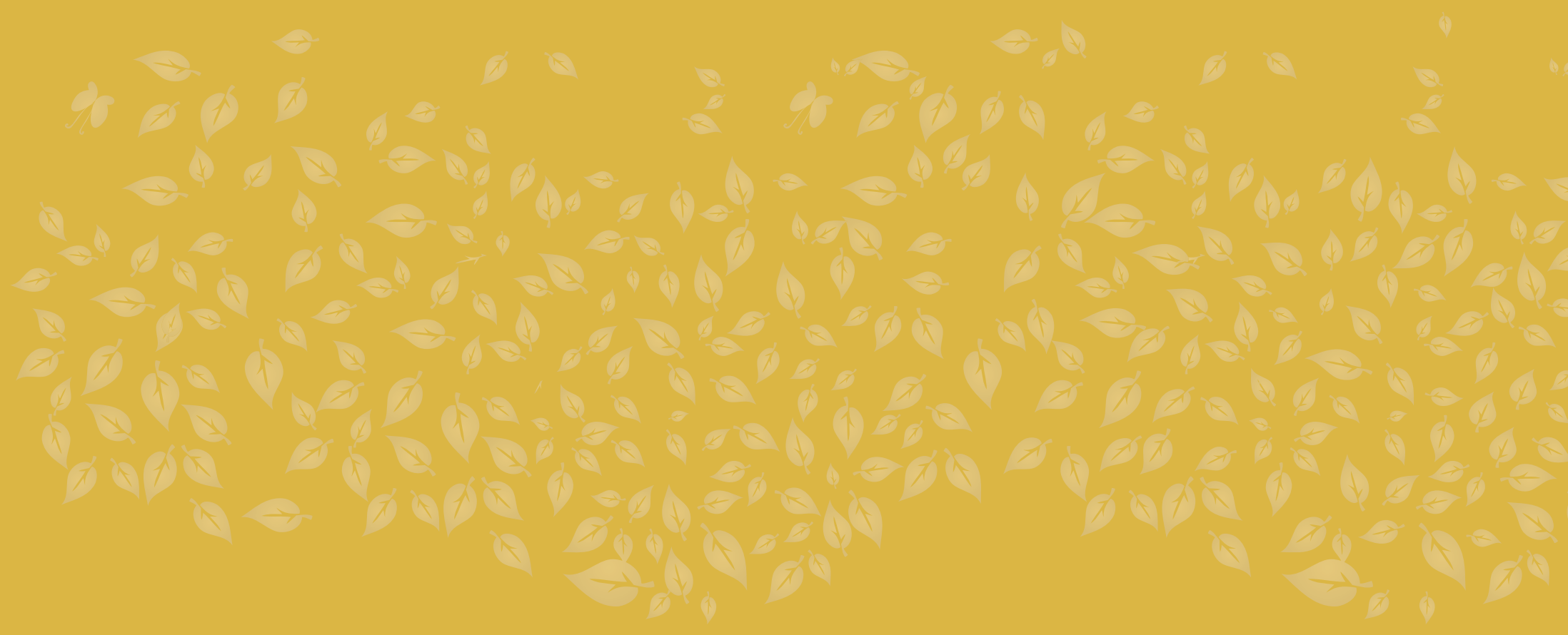

REV. COL. REH || Bogotá, Colombia || Volumen 10 || Página 46 - 58 || Noviembre 2011 || ISSN 1692 - 1879 